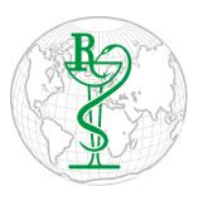

INDO GLOBAL JOURNAL OF

PHARMACEUTICAL SCIENCES

ISSN 2249- 1023

\title{
Viral Diseases, N-COVID-19, and Evolution: A Commentary
}

\author{
S.B. Ghosh $1,2^{*}$ \\ ${ }^{I}$ Department of Botany, Microbiology Laboratory, S. C. college, Habra, North 24 Parganas, West Bengal, India \\ ${ }^{2}$ Department of Microbiology, Bethune College, Kolkata, West Bengal, India
}

Address for Correspondence: S.B. Ghosh, sbghosh2010@msn.com

\begin{tabular}{l} 
Received: \\
28.06.2020 \\
Accepted: \\
10.11.2020 \\
Published: \\
21.12 .2020 \\
Keywords \\
Viral infections, \\
Genetic \\
Recombination, \\
Human genetic \\
bottleneck. \\
\hline
\end{tabular}

Received:

10.11.2020

Published:

21.12.2020

Keywords

Viral infections,

Recombination,

bottleneck.

\begin{abstract}
Viruses originated from 'Primordial Nucleic Acids' with lipid and protein encapsulations. Chance fusion with cellular entities and intracellular replication of exogenotes started the journey of viruses on this planet. While replicating, pairing occurred in between the viral exogenotes and the host endogenote at their homologous sites. Therefore, both of the genes become recombined. Sometimes the viral exogenotes get integrated into the host genome resulting in a new reading frame in gene sequences. So, at every cycle of cellular infection with a viral particle, both the host and the parasite genes are recombined. The virulent the viral infection, the better is the chance of gene recombination of the host. Every stratum of cellular living forms has its viral pathogen. In lower living forms, depending on the habitats, a complete gene or its fragments may enter a cell using membrane receptors from the nucleic acid pool resulted from the lysis of cellular entities or viral particles, and gene recombination occurs. These are Transforming Principles or Transposable Elements. This lateral passage of genes through individuals is called Horizontal Gene Transfer. When mediated by viruses, it is called Transduction. Eventually, gene recombination occurs at the cellular level. Genetic Recombination is the basis of speciation, survival, evolution, and a way out of genetic bottleneck in cellular life. Humans too are facing a genetic bottleneck and living on the edge of extinction. Therefore, the viral infections in humans might be one of mother nature's designs to find a way out of this genetic bottleneck and survive and evolve further. (c) 2020 iGlobal Research and Publishing Foundation. All rights reserved.
\end{abstract}

Cite this article as: Ghosh, S.B. Viral diseases, N-COVID-19, and evolution: a commentary. Indo Global J. Pharm. Sci., 2020; 10(2): 13-18. DOI: http://doi.org/10.35652/IGJPS.2020.10203 .

\section{INTRODUCTION}

This is time to review viral infections and diseases of all existing cellular living beings from another angle, with different perspectives and purposes. The foremost thing that needs to be conveyed here is to encourage people to get rid of the fear of death, social isolation, and mistrust. This is to evoke awareness in common readers that viral infection is a gift of Mother Nature to help humans and every other species to survive and evolve further. Viral diseases are not Mother Nature's fury or cruel revenge to kill and destroy humans and any other species evolved on earth. Mother Nature has designed the origin, survival, and the evolution of every species on the Planet Earth.

Every living species has the right to survive, to evolve, and overcome extinction from the face of the earth. Nevertheless, a species can only exist on earth by interacting with other species. Inter - and intraspecies interactive way of survival is a Mother Nature's design to let a species to evolve further. Of course, the demise of any single person is a matter of sadness in human society. Yet, when this article is read, people should know that those who have died because of any viral infections have contributed to humans' survival and evolution on Planet Earth. The departed souls are bid adieu with respect and be treated as martyrs.

Humans are standing at a turning point in the passage of immortal time that asks the questions on possibilities and consequences of human existence on this Planet Earth. The Planet Earth, called the "Blue Planet", where 'Millions of Cellular Living Species with Billions of Individuals' are known to exist, is probably the only planet with cellular lives in our knowledge to date, in the whole Milky Way Galaxy (MWG) [1,2]. In terms of cellular life, it then becomes 'the Lonely Planet', amongst the 100 billion probable planets, in one of $100-400$ billion Solar Systems in MWG, where our 


\section{Indo Global Journal of Pharmaceutical Sciences, 2020; 10(2): 13-18}

Solar System is revolving around the Milky Way in the farthest corner of one of its spiral arms, called Orion Spur $[3,4]$.

Thereby, the origin and survival of the 'Intelligent or Wise Humans', the Homo sapiens Linn. [5] (In Latin Homo is human \& sapiens is intelligent/wise), on this lonely planet, must have been another 'lonely event' in the Celestial History of MWG. Modern human, the Homo sapiens sapiens, is a subspecies of the extant Homo sapiens Linn. Intelligent humans now stand alone on this lonely planet. In an approximately 2.2 million Light Year Area of the MWG, without any close relatives of Homininan Humans on earth or any other planet nearby, modern humans are standing alone with a 'Genetic Bottleneck' [6,7], without any 'natural gene reservoir' in near sight to have any chance to exchange their genes with and recombine them.

Three prime factors are now pushing humans towards the edge of extinction. The first one is obviously the Human' Genomic Bottleneck' in the absence of any closely related Hominid subspecies. The second is the 'Y-chromosomal Bottleneck' in the absence of any homologous counterpart in the human cells. Since the Neolithic Period, around $10-7$ Kya (kilo year ago), aggressiveness in human males can now be correlated to their Y-chromosomal bottleneck [8,9]. The third is the Population Explosion, which is out of context in this perspective commentary.

Only time can tell the Nature and degree of ferocities of these factors. However, the Genetic Bottleneck of Humans forms the basis of this article. Without any doubt, humans need to think and rethink about COVID-19, SARI-I, SARI-II, SARS, MERS, Bird Flu, Swine Flu, Bovine Flu, Ebola, Dengue, Hepatitis, AIDS etc. [10] and every other deadly viral disease of humans and other living beings. Humans need to develop the most effective vaccines. We need to find out potent antiviral drugs and cheaper and more efficient diagnostic 'kits'. Although in the end, no drug, no medicine, and no vaccine can rescue humans from their 'genetic bottleneck' and save them from an 'Extinction Holocaust'.

Humans need to know why these viruses are there in Nature and what precisely these viruses are doing in all our living forms and habitats. It is now very well known that there cannot be anything for nothing in Mother Nature's inventory. Apart from a clinical, medical, and pathological point of view, the viral infections are radically different from bacterial, fungal, and other infectious diseases when looked into it from a genetic point of view.
Viruses interact directly with the host's genome at every new infective cycle and Horizontally or Laterally transfer, transport, and eventually recombine genes of both the host and the viral parasite itself while passing horizontally or laterally through a wide variety of related and unrelated host species. As viruses do have a wide range of alternative host species, they play a significant role in the evolution of the host species $[11,12]$. Therefore, the human genetic bottlenecks can only be rectified or reverted back with the help of these 'Genetic Vectors' called viruses.

Since Charles Darwin's "Origin of Species" and "Natural Selection and Survival of the Fittest", it is well known that these diseases and natural calamities are Mother Nature's 'selection tools' to select the 'fittest' and let them continue to survive and evolve. Viruses are our last hope to survive from extinction and evolve further. Humans need to understand this enormous gift of Mother Nature, and sooner the better. Kat Arney commented "Viruses give us infections from the common cold to COVID-19 and AIDS. But research shows that they may also have played a key role in shaping the evolution of Homo sapiens".

The explanatory answers are the bases of this topic that have been narrated sequentially in the following

\section{THE PERSPECTIVE COMMENTARY AND EXPLANATORY NOTES}

1. How, where, and when did these viruses originate in Nature? There are as many hypotheses as experimental proofs to answer these questions.

2. About 700 - 600 mya (million years ago), at the time of origin of fully functional and replicating cellular life, viruses originated from 'Primordial Nucleic Acids or NA (NA here means either of the Ribonucleic Acid or RNA and Deoxyribonucleic Acid or DNA)' with lipid and protein encapsulations $[13,14]$.

3. Unlike cellular NA, the survival and replicability of these early 'encapsulated' viral NA were at stake due to lack of necessary enzymes within these early 'pre-viral capsules', required for harnessing the energy and producing building block materials.

4. Chance fusion with cellular entities, as obviously the lipid coat would often do, started the journey of these 'exogenotes (the NA external to the cellular NA or endogenotes) of pre-viral origin' on this planet. However, the interactions between modern viruses and their hosts are based upon membrane-bound receptors [15].

5. The killing of early cellular life (hosts) with these early viral forms could not be an objective in Mother Nature's inventory. 


\section{Indo Global Journal of Pharmaceutical Sciences, 2020; 10(2): 13-18}

6. Viral exogenotes, along with 'Capsids' (viral protein coat or sometimes lipo-protein envelops - quite different from the lipoprotein membrane envelops of present-day animal/human viruses), replicated inside these early cellular hosts with the help of the host's enzymes and remained inside the cellular envelops till the death these host cells. Virion (viral individuals) could have been released only at the death and lysis of host cells. And thus, the journey of viruses began at about 400 mya, alongside the evolutionary journey of 'cellular living entities' [16].

7. The viruses evolved as an 'Obligate Parasite' as they could not replicate outside the cellular environment. However, since their origin to date, viruses can exist and survive outside cell in the form of crystalline or particulate matter in all geospheres on earth.

8. Some very interesting and obvious things happened during these early stages of 'Evolutionary Developments' of viruses. The 'Viral Exogenotes' started pairing with the host's 'homologous (same or very similar)' NA segments, got integrated into the host's NA (endogenote) and the 'host-virus combinations (endogenotes-exogenotes combines)' developed devices (necessary enzymes - most likely the 'Ribozymes' the same way 'Lariats' are loops out and eventually are excluded during 'Gene Splicing') to get the viral NA liberated from the host's NA or host-genome [17].

9. Active release of mature Virion into the environment (still aqueous, most likely) had been made possible with the development' Reverse Pinocytosis' in these early cellular environments.

10. Now the most vital question arises here, after point No.1. Why did mother nature let viruses evolve?

11. The answers are many and are based upon dozens of hypotheses and hundreds of experimental proofs. At the time of the release of viral NA from the host's NA (referred to points $6 \& 7$ ), there had been, most of times, a portion of the host's NA remaining attached to the viral NA [18].

12. An interesting thing happened at the same time. While releasing the viral NA from host-genome, a portion of viral genome or NA also remained permanently attached to the host NA.

13. It is now amazing to note that when the newly formed viral NA and the host NA are read from end to end, it is altogether a new 'reading frame' in both the recombined viral genome and the recombined host genome.

14. While fusing with or attacking the likely and similar host cells, these new recombined viruses characteristically might have been remaining the same and similar, or, could be more potent, or, might have lost their potency altogether.

15. Another possibility evolved at the same time that is the total or partial destruction of viral NA or viral genome inside the host cell by the host's enzymes.
16. Death and lysis of cellular and viral entities started creating an NA-pool around these early cellular lives' living environment.

17. It was found out that the cellular living entities, since the very early stages of their development and evolution, developed structures on their lipid-protein envelops (the cell membranes) to take up tiny (or suitable) NA - fragments from these NA - pool around them.

18. Cellular NA or cellular genome started interacting with these foreign or exogenomic NA taken up from the pool when they are homologous or similar. These external NA, when integrated into the cellular genomic NA, gave rise to a new recombined cellular genome.

19. The new recombined cellular generation might have been very suitable or 'Fit' to evolve further 'progressively', or, the new Recombination might have been 'less-suitable or lessfit' and evolved 'regressively', or, altogether might have been 'very unfit' and thus became extinct. This process of selecting the potentiality or fitness of the evolving recombined genomes is now known as "Natural Selection".

20. One essential thing we need to recall at this moment is the absence of sexual reproductive mechanisms to recombine cellular genomes during the early stages of origin and evolution of cellular life.

21. The mechanism of genomic Recombination through true sexual reproduction (while keeping in mind the 'Bacterial Conjugation') evolved much later in the evolutionary time scale when higher forms of eukaryotic cellular life started emerging and evolving.

22. Therefore, during these early stages of cellular origin and evolution, Mother Nature devised four different, yet interacting, mechanisms to recombine and reorganize cellular genomes to give 'Life' a chance to evolve and progress further absence of true sexual reproduction.

22.1. Release of NA fragments from the cellular genome itself, movement of these fragments within the intracellular space and getting these fragments intercalated and lodged at some different locations within the cellular genome. So, the genes wear a new look. These fragments are now called 'Transposons' or 'Transposable Elements'.

22.2. Interaction, intercalation, and incorporation of exogenotes into the main-frame genomic sequences, following their 'intake' from the NA-pool present in the surroundings. Here, the exogenomic NA fragments are called 'Transforming Principles' and the phenomenon of recombination is known as 'Transformation'.

22.3. Recombination of the cellular genome through viruses, called 'Vectors', has already been stated above. Here the viral genome acts as the 'Transducing 


\section{Indo Global Journal of Pharmaceutical Sciences, 2020; 10(2): 13-18}

Elements' and the phenomenon is now known as 'Transduction'.

22.4. The fourth mechanism is changing the NA-base pairs in the main-frame genome by the effect of some Chemical (numerous categories Organic and Inorganic compounds) or Physical Agents (e.g. various ionizing \& non-ionizing rays), called 'Mutagens'. This process is called 'Mutation'.

23. In all these cases (No. 22 i-iv) 'the Cellular Genetic Reading Frame' (the 'Cleartext' in scientific jargon) is changed and is called 'Recombined'. It is amazing to note that these asexual gene-recombining processes (point No. 22 i-iv) are still active in full form as they evolved and can affect an individual at any stage of its Life Cycle (or Life History).

24. Logical suppositions tell us that these processes shall remain unaltered as long as there will be cellular life on earth. Genetic Recombination of cellular lives or species is Mother Nature's basic design to let new species to originate and to allow evolution to continue.

25. When this recombined NA or genome is directly passed onto the 'daughter cells' or offspring, the phenomenon is termed as 'Vertical Transmission of Genome (or Traits, or, Characters in Mendelian Principles) to Filial Generations (from Latin Filia daughter \& Filius son)'.

26. Whereas, the processes of gene duplication, gene addition, gene deletion and gene recombination through 'Transforming Principles' and 'Transducing Elements' is called Horizontal Transmission of Gene, or Lateral Gene Transfer, or Horizontal Movement of Genetic Materials and finally can be viewed as 'Mother Nature's Genetic Engineering. Furthermore, the Transposable Elements or Transposons are also, sometimes, transferred horizontally.

27. Terrestrial viruses evolved with the origin of terrestrial forms of cellular lives about 540 mya.

28. Most probably, the animal viruses of terrestrial habitat evolved with insects' origin at about 450 - 400 mya.

29. Amphibian and Reptilian viruses appeared about 360 -300 mya.

30. Avian and Mammalian viruses came into being at about 150 - 130 mya.

31. Long before the origin of Primates at about 65 mya, animal viruses found their refuge in numerous alternative 'host - lines' of Terrestrial Chordates, cutting across the 'Class Lines' of Amphibia, Reptilia, Avis, and Arthropoda (Sub Cl. Insecta in particular), and finally crossing Mammalian barrier $[19,20]$.

32. Therefore, the Origin of Hominid Humans, approximately about $2.5-2.0$ mya, was associated with the viruses already in them.

33. Sexual reproduction is a tool for recombining the Intra-Species genome i.e. the genome of the entire range of any particular species. With the origin of 'sexual reproduction', the vertical passage of recombined genome onto the filial generations was ensured. However, the future course of these filial generations was always subjected to 'Natural Selection'.

34. In diploid living forms (the $2 n$ state - every cell with two sets of homologous chromosomes) where for haploid gamete formation (the $\mathrm{n}$ state - gametes get only one set of homologous chromosomes from the pair), the homologous pairs exchange and recombine their chromosomal segments and so the genes, during Meiosis (called meiotic cell division or reduction division where homologous pairs are separated and only one of them goes to the daughter cell forming haploid gamete) through 'crossing-over'. These gametes unite (fusion) to form zygote and the diploid forms are reinstated and continued.

35. In haploid living forms, the gametes are produced through mitotic cell division (vegetative or conservative cell division) and on fusion, the diploid (2n) zygote is formed in the same and similar way as mentioned in point No. 28. Then, sooner or later, meiosis occurs in the zygotic cells, chromosomal segments or genes are recombined through crossing over, homologous pairs are separated, and the journey of haploid individuals begins.

36. Therefore, in sexually reproducing species, whether the free-living individuals are diploids or haploids, there is essentially a meiotic cell division stage where and when the genes get recombined.

37. When we look at 'Human Genome', all the Anthropogenic Sub-groups of HOMO SAPIENS SAPIENS (L1, L2, L3, etc.), Sub-sub-groups (M, N, O etc.) and Mitochondrial and Y-chromosomal haplo-groups appear same and similar.

38. Human Genome (as a whole) already has arrived at a genetic 'Bottleneck' which means all possible Recombination of genes had taken place and recombinant (having new look recombined genome) individuals have been tested, exhausted, and delimited. The absence of any closely related Hominid sub-species and variety, which could replenish and recombine human genome to thrive competently in the man-made fastchanging environment of our modern times, have thereby delimited further the chance of getting new genome segments.

39. Although our genome is approximately $95-96 \%$ similar to apes, we do not have a way to recombine with them. This is called 'interspecies incompatibility' [21].

40. With a second look at the Human Genome, another amazing fact is found. There are many genes in the human genome that have 'horizontally been transmitted' in course of time via vectors, i.e. viruses (e.g. about 8\% Endogenous Retroviral Genes, human Oncogene, some new flu genes, some new enzymes that are absent in apes, etc.) and having 


\section{Indo Global Journal of Pharmaceutical Sciences, 2020; 10(2): 13-18}

been being integrated, these genes of viral origin have eventually become part of the human genome [22].

41. Being far ahead and far away from HOMO ERECTUS, evolution of Modern Man (HOMO SAPIENS), since Last Ice Age (1.6 mya approx.), began with complete extinction (man-made?) of most closely related Homo species i.e., Homo NEANDERTHALENSIS.

42. Therefore, today's modern human species (HOMO SAPIENS SAPIENS) is living dangerously on the edge of extinction, with no animal species near sight that can contribute to recombine human genome it a new look.

43. Even if humans manage to survive for another five to ten thousand years in this present genomic form, this sexually reproducing unisexual humans may have to forego with their 'maleness' because of the fact that their 'Ychromosome', the carrier of the maleness, has no homologous counterpart in their diploid set of 23 pairs of chromosomes. As such, the Y-chromosome has become unable to recombine through 'crossing-over' with any other chromosome of their genome.

44. We cannot foresee the future of human evolution in the absence of maleness in them. There is a saying: "life always finds a way" (taken from the Jurassic Park movie), which might be true for humans.

45. Mother nature might have devised viruses to replenish, realign, rejuvenate and recombine genes across the closely and distantly related species lines of humans, and realign and recombine genes intracellularly, irrespective of homologous and heterologous chromosomes, to overcome genetic bottleneck.

46. Then these gene transporting (transducing) viruses would surely need deceptive devices to bypass the mammalian defensive immune system, so as to go inside the cell and unload the genes. N-COVID-19 is doing just that with their new lipo-protein receptor spikes on their capsids to bind with ACE-2 or HAP-2 like cellular receptors and is being taken inside by the human cells themselves.

47. Thereby, the N-COVID-19 virus is escaping the bindings with TCRs (T-cell Receptors), BCRs (B-cell Receptors), MHC-I, and MHC-II (MHC is Major Histocompatibility Complex and I \& II are variants) like membrane-bound protein receptors of Mammalian-ImmuneActive-Cellular-Systems which are mainly responsible for 'Antigen Presentation' to the 'Antibody Producing B-Cells' and the 'Killer Tc-cells' (Tc-cells are Cytotoxic T-Cells which kills the Antigen Bearing or Antigen Containing cells in mammalian systems). Therefore, the primary immune response is compromised or jeopardized, the development of 'Herd Immunity' (total immunity of any particular community) is delayed and disease symptoms develop as the viruses can now grow within the body cells. However, with time, while the COVI-19 is replicating within the human or other mammalian hosts, a huge quantity of fragments of lipoprotein capsids of COVID-19 become available within the hosts and are captured by those TCRs, BCRs, and MHCs, and are presented to the antibody-producing B-cells. The antibodies, which are active against the N-COVID-19 capsid-complex, are then produced. The COVID-19 virus particles are arrested by these antibodies and finally the desired 'Herd Immunity' is developed.

48. Therefore, while these N-COVID-19 viruses are spreading in a cyclic order through human-animal (suspected bats, pangolins, etc.)chain, they are carrying with them the parts of the host's genes at the end of a cycle and recombining these genes with host's genes at their next infective cycles. The same is true for all the viruses.

49. Maybe, every viral infection in humans (of course in other living forms too) and subsequent development of 'herd immunity', is an effort of Mother Nature to rescue humans from their genetic bottlenecks and their eventual extinction.

50. Nature should be respected. Viral infections are not a curse of Nature. It is Mother Nature's 'blessing in disguise'. Nobody has the right to destroy any of her creations. Nothing can play against Mother Nature's will.

\section{CONCLUSION}

All effort is needed against every new viral attack to find new and potent testing kits, antiviral drugs, and most suitable vaccines. There is no question about it. Simultaneously, humans should get themselves rid of frustrations and fear psychosis of death due to viral pandemics. It becomes a matter of happiness to know that due to these viruses, new human generations with their genomes recombined are in the offing and the present human society has contributed positively to further human evolution to get rid of the 'human - genetic bottleneck'. Viruses are the last ray of hope for humans, and every other evolving species on earth, to evolve further before the sun finally sets forever in another 500 million years.

\section{CONFLICTS OF INTEREST}

The author confirms that this article content has no conflict of interest.

\section{ACKNOWLEDGEMENT}

The author acknowledges the inspirational guidance of Dr. Supratik Kar, Jackson State University, Jackson, Mississippi, USA for completion of this article. Continuous encouragement and enthusiasm from Dr. Kar was an impetus to accomplish the work. This article is being dedicated to my family members without whose support and encouragement the writing could not have been made possible. 


\section{Indo Global Journal of Pharmaceutical Sciences, 2020; 10(2): 13-18}

\section{REFERENCES}

1. Gupta A, Mathur S, Krongold Y, Nicastro F, Galeazzi M. A Huge Reservoir of Ionized Gas Around the Milky Way: Accounting for the Missing Mass?. ApJ. 2012;756: L8.

2. Gibney E. Earth's new address: 'Solar System, Milky Way, Laniakea'. Nature. 2014.

3. Kafle PR, Sharma S, Lewis GF, Bland-Hawthorn J. Kinematics of the Stellar Halo and the Mass Distribution of the Milky Way Using Blue Horizontal Branch Stars. ApJ. 2012;761:17.

4. Kafle PR, Sharma S, Lewis GF, Bland-Hawthorn J. On the Shoulders of Giants: Properties of the Stellar Halo and the Milky Way Mass Distribution. ApJ. 2014;794:17.

5. Rogers AR, Mackay A, Arnold ML. The Hybrid Origin of "Modern" Humans". Evolution Biol. 2015;43:1-11.

6. Ambrose SH. Late Pleistocene human population bottlenecks, volcanic winter, and differentiation of modern humans. J Hum Evol. 1998;34:623-651.

7. Zielinski GA, Mayewski PA, Meeker LD, Whitlow S, Twickler MS, Taylor K. Potential Atmospheric Impact of the Toba Mega-Eruption 71,000 years ago. Geophys Res Lett. 1996;23:837840 .

8. Mendez F, Krahn T, Schrack B, Krahn A-M, Veeramah KR, Woerner AE, Fomine FLM, Bradman N, Thomas MG, Karafet TM, Hammer MF. An African American paternal lineage adds an extremely ancient root to the human Y chromosome phylogenetic tree. Am J Hum Genet. 2013;92:454-459.

9. Kamin M, Saag L, Vicente M, et al. A recent bottleneck of Y chromosome diversity coincides with a global change in culture. Genome Res. 2015;25: 459-466.

10. Slonczewski JL, foster JW. In: Microbiology: An Evolving Science. Betsy Twitchell (Ed). W. W. Norton \& Company, New York. 2014.

11. Robinson KM, Sieber KB, Dunning Hotopp JC. A review of bacteria-animal lateral gene transfer may inform our understanding of diseases like cancer. PLoS Genet. 2013;9: e1003877.

12. Acuña R, Padilla BE, Flórez-Ramos CP, Rubio JD, Herrera JC, Benavides P, Lee S-J, Yeats TH., Egan AN., Doyle JJ., Rose JKC. Adaptive horizontal transfer of a bacterial gene to an invasive insect pest of coffee. PNAS. 2012;109:4197-4202.

13. Krupovic M, Dolja VV, Koonin EV. Origin of viruses: primordial replicators recruiting capsids from hosts. Nat Rev Microbiol. 2019;17:449-458.

14. Forterre P, Prangishvili D. The major role of viruses in cellular evolution: facts and hypotheses. Curr Opin Virol. 2013;3:558-565.

15. Maginnis MS. Virus-receptor interactions: the key to cellular invasion. J. Mol. Biol. 2018;430:2590-2611.

16. Sabath N, Wagner A, Karlin D. Evolution of viral proteins originated de novo by overprinting. Mol Biol Evol. 2012;29:37673780 .

17. Venkataraman S, Prasad B, Selvarajan R. RNA dependent RNA polymerases: insights from structure, function and evolution. Viruses. 2018;10:76.
18. Koonin EV. Viruses and mobile elements as drivers of evolutionary transitions. Philos Trans R Soc B Biol Sci. 2016;371:20150442.

19. Lancet D, Zidovetzki R, Markovitch O. Systems protobiology: origin of life in lipid catalytic networks. J R Soc Interface. 2018;15:20180159.

20. Terasaka N, Azuma Y, Hilvert D. Laboratory evolution of virus-like nucleocapsids from nonviral protein cages. Proc Natl Acad Sci. USA. 2018;115:5432-5437.

21. Auton A, Abecasis G, Altshuler D. et al. A global reference for human genetic variation. Nature. 2015:526: 68-74.

22. Suntsova M, Garazha A, Ivanova A, Kaminsky D, Zhavoronkov A, Buzdin A. Molecular functions of human endogenous retroviruses in health and disease. Cell Mol Life Sci. 2015;72:3653-3675. 